\title{
PARTIAL TRANSMIT SEQUENCE (PTS) TECHNIQUE TO REDUCE THE PEAK TO AVERAGE POWER RATIO (PAPR) IN OFDM SYSTEM
}

\author{
Amrutha p $\mathbf{p}^{1}$, Anandswamy A $\mathbf{S}^{2}$ \\ ${ }^{1} M$-Tech Student, Department of ECE, TJIT, Bengaluru, Karnataka, India \\ ${ }^{2}$ Associate Professor, Department of ECE, TJIT, Bengaluru, Karnataka, India
}

\begin{abstract}
In this paper, partial transmit sequence (PTS) technique is used to improve the peak to average power ratio (PAPR) statistics in orthogonal frequency division multiplexing (OFDM) system. The PAPR value should be less than or equal to $5 d b$, but the PAPR value of OFDM signal is $12 \mathrm{db}$ which is one of the major drawback. To reduce the PAPR in OFDM system we are using PTS technique but the drawback of PTS is that it has high computational complexity. Hence in this paper, we propose two modified low complexity PTS methods called serial PTS (S-PTS) and parallel PTS (P-PTS) to reduce the computational complexity of PTS technique based on the weighting factors chosen from the code words of linear block codes.
\end{abstract}

Keywords: PAPR, PTS, OFDM

\section{INTRODUCTION}

OFDM is widely used in modulation and multiplexing technology. It is one of the modulation techniques that transmit signals through multiple carriers. The main motivation to apply OFDM is to enhance the robustness against the narrow band interference. In single carrier system if signal get interfered then entire relationship gets failed where as in multicarrier system only a small proportion of the sub carrier will be affected. The main advantages of OFDM system is high spectral efficiency as compared to double side band modulation scheme, computationally efficient by using FFT techniques to implement the modulation and demodulation functions, provides high data rate of about $6 \mathrm{Mbps}$ to $48 \mathrm{Mbps}$ and it supports different modulation scheme like quadrature amplitude modulation (16QAM and 64QAM), binary phase shift keying (BPSK) and quadrature phase shift keying (QPSK) based on channel conditions. The major exertion in OFDM system is raise in peak to average power ratio (PAPR) which leads to various distortions like amplitude, frequency and inters symbol interference in the transmitter chain and degradation of system performance i.e. bit error rate (BER). Also requires high linear power amplifiers which increase the cost of the system. To overcome this problem we are using PTS technique. In this paper the partial transmit sequence (PTS) scheme is the most efficient method for reducing PAPR compared to other techniques like clipping and filtering, selective line mapping tone reservation, interleaving, peak windowing etc.

There are two modified PTS methods like serial PTS (SPTS) and parallel PTS (P-PTS) to reduce the computational complexity of PTS technique based on the weighting factors chosen from the code words of linear block codes and $\mathrm{BCH}$ codes and also in order to still reduce the PAPR values of designed system.

\section{PROBLEM STATEMENT}

\section{A. PAPR}

PAPR is a significant characteristic of communication scheme. A low PAPR allows the transmit power amplifier to function efficiently, where as a high PAPR forces the transmit power amplifier to have a huge back off in order to ensure linear amplification of the signal. An OFDM base band signal y ( $\mathrm{n}$ ) of $\mathrm{N}$ subcarriers can be expressed as

$$
\mathrm{y}(\mathrm{n})=1 / \mathrm{N} \sum_{K=0}^{N-1} \mathrm{Y}_{\mathrm{K}} \exp (\mathrm{j} 2 \pi \mathrm{nK} / \mathrm{N})
$$

The PAPR of OFDM system is the ratio of peak power (peak amplitude squared) to the average power (RMS value squared). The PAPR in db is given by

$$
\text { PAPR }=\frac{\max |y(n)|^{2}}{E\left[|y(n)|^{2}\right]}
$$

Where, $\mathrm{E}$ [.] is expected value, $\mathrm{y}(\mathrm{n})$ is the time domain data obtained by IFFT and Yk is the frequency domain data.

The PAPR is the relative between the maximum powers of a section in a given OFDM transmit symbols alienated by the typical power of that OFDM symbol. PAPR occurs when in a multi-carrier scheme the diverse sub-carriers are out of period with each other. At each moment they are distinctive concerning each other at distinctive stage values. When every one of the focuses accomplish the greatest esteem at the same time; this will bring about the yield envelope to all of a sudden shoot up which causes a crest in the yield envelope. Because of presence of substantial number of freely regulated sub-carriers in an OFDM framework, the crest estimation of the framework can be high when contrasted with the normal of the entirety framework. 


\section{b. PARTIAL TRANSMit SEQUence (PTS)} TECHNIQUE

PTS is one of the PAPR reduction techniques. The main design of this scheme is the data block is divided into non overlapping sub block with independent rotation factor. This method divides the frequency vector transmitter signal into smaller blocks before applying the phase transformations. Here phases are randomly generated and the generated phases are multiplied. Thus by modifying the data by using the phase manipulations, the PAPR value will be reduced. If there is more PAPR value, high power amplifiers are required. So by reducing the PAPR by using this technique, low power amplifiers are sufficient to design the system which results in decreasing the cost of the design. In PTS method the frequency vector is divided into smaller blocks before applying the phase transformation and the main advantage of PTS technique is it has lower PAPR and less redundancy.

In the PTS technique the input data $\mathrm{Y}$ is partitioned into $\mathrm{R}$ disjoint sub blocks,

$\mathrm{Yr}=[\mathrm{Yr}, 0, \mathrm{Yr}, 1 \ldots . \mathrm{Yr}, \mathrm{N}-1] \mathrm{r}=1,2 \ldots \ldots . \mathrm{R}$ such that

$\sum_{m=1}^{M} \mathrm{Yr}=\mathrm{Y}$ and the sub block are combined to minimize the

PAPR in the time domain. The L-times over sampled time domain signal of $\mathrm{Yr}, \mathrm{r}=1,2 \ldots \mathrm{R}$ is denoted as $\mathrm{yr}=[\mathrm{yr}, 0$, $\mathrm{yr}, 1 \ldots \ldots \ldots \ldots . . . . \mathrm{yr}, \mathrm{NL}-1] \mathrm{r}=1,2, \ldots \ldots \mathrm{R}$ is obtained by taking IFFT of length NL on Xm concatenated with (L-1) N zeros. These are called the partial transit sequences. The complex phase factor $b r=\exp (j \emptyset r) r=1,2 \ldots R$.

$$
\overline{\mathrm{y}(\mathrm{n})}=\sum_{n=0}^{N-1} \operatorname{br} \mathrm{y}(\mathrm{n})
$$

The phase factors are chosen based on $R$ value if $R=4$ then the phase factors are $\{1,-1\}$ and for $\mathrm{R}=16$ the phase factors are $\{1,-1, \mathrm{j},-\mathrm{j}\}$. After applying PTS algorithm we plot the graph of PAPR in $\mathrm{db}$ versus CCDF.

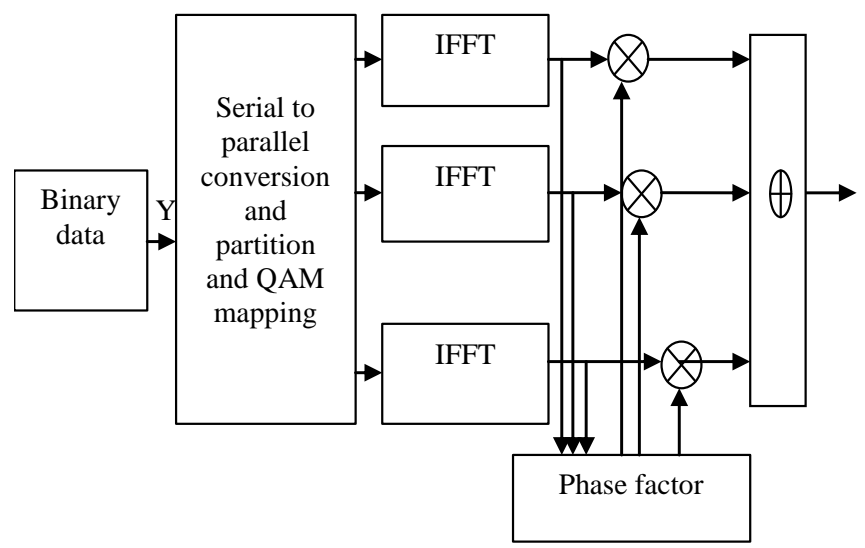

Fig.1. Block diagram of PTS

\section{PARALlEL AND SERIAL PTS METHOD}

Parallel and serial PTS method is used to reduce the computational complexity of PTS technique and provides error correction capability for the weighting factors.

In parallel partial transmit sequence (P-PTS) the code words are generated from the $\mathrm{BCH}$ codes and golay codes are simply considered as weighting factors. The code words are denoted as $\mathrm{g}(\mathrm{y})$, where $\mathrm{g}(\mathrm{y})$ is the generator polynomial for parallel PTS given by,

$\mathrm{Gp}=\left(\begin{array}{l}\mathrm{g} 1(\mathrm{y}) \\ \mathrm{g} 2(\mathrm{y}) \\ \mathrm{gk}(\mathrm{y})\end{array}\right)$

In serial partial transmit sequence (S-PTS) the consecutive code words are added to generate the modified code words generated from the $\mathrm{BCH}$ codes and golay codes and then are considered as weighting factors. $\mathrm{g}(\mathrm{x})$ is the generator polynomial for serial PTS given by

$$
\mathrm{Gs}=\left(\begin{array}{c}
\mathrm{g} 1(\mathrm{y}) \\
\mathrm{g} 1(\mathrm{y})+\mathrm{g} 2(\mathrm{y}) \\
\mathrm{g} 2(\mathrm{y})+\mathrm{g} 3(\mathrm{y}) \\
\\
\mathrm{gk}-1(\mathrm{y})+\mathrm{gk}(\mathrm{y})
\end{array}\right)
$$

$\mathrm{G}_{\mathrm{p}}$ and $\mathrm{G}_{\mathrm{s}}$ is $\mathrm{K} \times \mathrm{N}$ matrix. The weighting factors of P-PTS and S-PTS are from the code words of $\mathrm{BCH}(15,11,3)$, $\mathrm{BCH}(15,7,5), \mathrm{BCH}(15,5,7)$ and $(24,12,8)$ golay code.

\section{METHODOLOGY}

In OFDM system peak to average power ratio is the major drawback to overcome this problem we are introducing the partial transmit sequence technique to reduce the peak to average power ratio (PAPR). To reduce PAPR there are many techniques like clipping and filtering, selective line mapping, peak windowing, scrambling and block coding but this techniques have some disadvantages like

- Causes interference/distortion which degrades the system performance. Filtering after clipping reduce distortion but may also cause some peak re-growth.

- Complexity issue as selective line mapping scheme needs numerous IFFT operations.

- In peak windowing technique bit error rate and out of band radiation is increased.

- Scrambling technique gives reduced performance when number of sub carriers increased 


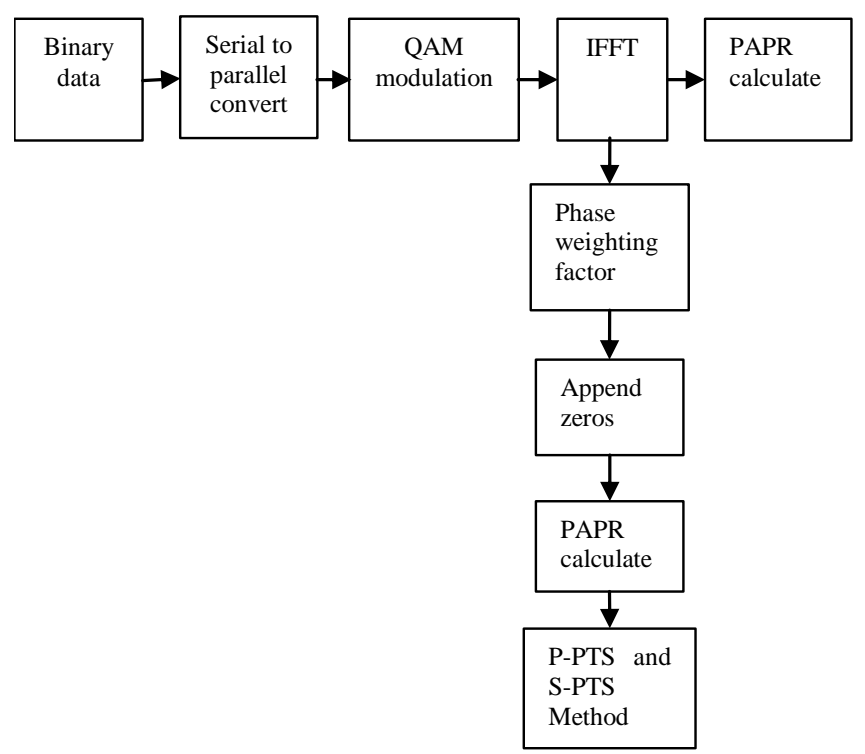

Fig.2. The proposed system

\section{E. PROCESS FLOW}

Step 1: An input image is taken.

Step 2: Image is converted to binary data.

Step 3: The binary data is converted from serial to parallel.

Step 4: Defining the modulation array value $M$ and modulation scheme to be performed.

Step 5: Reshaping the binary data into group of bits to perform QAM modulation.

Step 6: Define the modulator function and number of points in the IFFT block.

Step 7: After the modulation operation, the data is divided into frames. For each frame, IFFT operation is applied to generate the OFDM data and PAPR is calculated.

Step 8: For Reducing the PAPR, we are modifying the phases of the modulated data. Then we are applying the IFFT operation and PAPR is calculated. The PAPR calculated after the phase change operation is found to be less than the original OFDM signal.

Step 9: Generate the various combinations of the phase values based on $M$, of $M=4$ we are considering the phase values as $[1,-1]$ and if $M=16$ the phase values are $[1-11 j$ $1 \mathrm{j}]$.

Step 10: Divide the modulated data into four disjoint sets. For each of the disjoint sets, append zeros to make the data same as original data.

Step 11: Then one of the phase vectors is selected from the previously generated phase values. Then each set obtained above is multiplied by each of the phase values.
Step12: Then all the vectors are added to generate a single vector. For this vector IFFT operation is applied, and PAPR is calculated. This PAPR is compared with previously calculated PAPR.IF this PAPR is reduced, and then this modified OFDM signal is considered for the transmission.

Step 13: Steps 10, 11 and 12 are repeated for the phase vectors and OFDM signal with minimum PAPR is selected for transmission. Along with the OFDM data selected, the respective phase vector is also transmitted. Then PAPR v/s $\mathrm{CCDF}$ (complementary cumulative distribution function) is plotted.

Step 14: Parallel and serial PTS is used to reduce the computational complexity of partial transmit sequence based on the fact that the weighting factors are chosen as code words of linear block code.

\section{SIMULATION RESULTS}

This section of the given paper provides the simulation of expected results. Here we plot the graph of PAPR in $d b$ versus CCDF.

CCDF (complimentary cumulative distribution function) form a time domain signal. The CCDF curve shows the amount of time a signal spends above the average power level of the measured signal or equivalently the probability that the signal power will be above the average power level. It is a statistical power calculation method and can only be performed on time domain data. This measurement provides more insight of instantaneous power across the time. CCDF curve will have power in $\mathrm{db}$ on $\mathrm{X}$ axis and units of percentage on Y axis.

$\mathrm{CCDF}=\operatorname{Pr}\left(\mathrm{PAPR}>\mathrm{PAPR}_{\mathrm{o}}\right)$

\section{A. PAPR of OFDM System}

The peak to average power ratio of OFDM system is found to be $20 \mathrm{db}$.

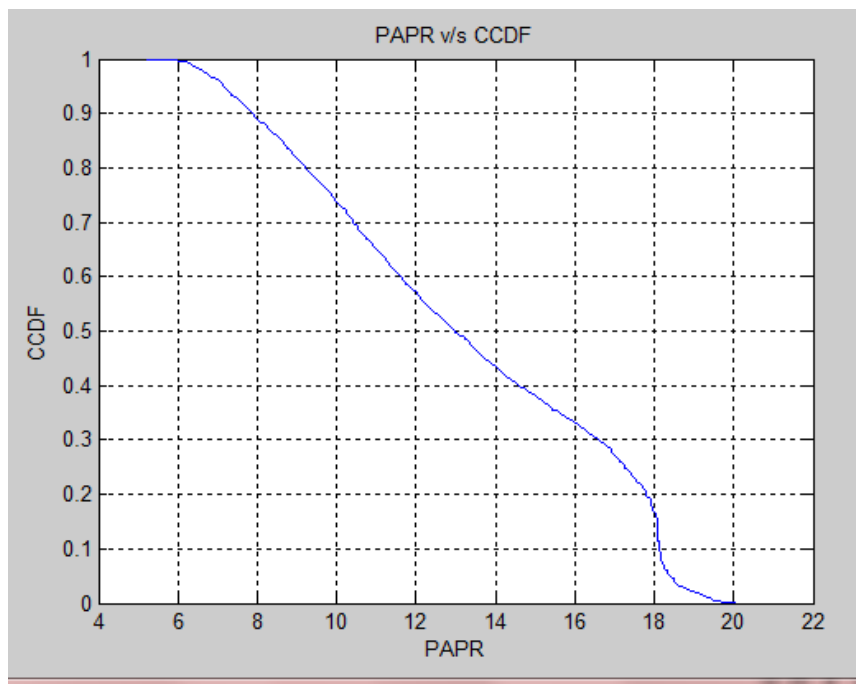




\section{B. PAPR by applying PTS algorithm}

The PAPR of OFDM system after applying partial transmit sequence algorithm is reduced to $7 \mathrm{db}$.

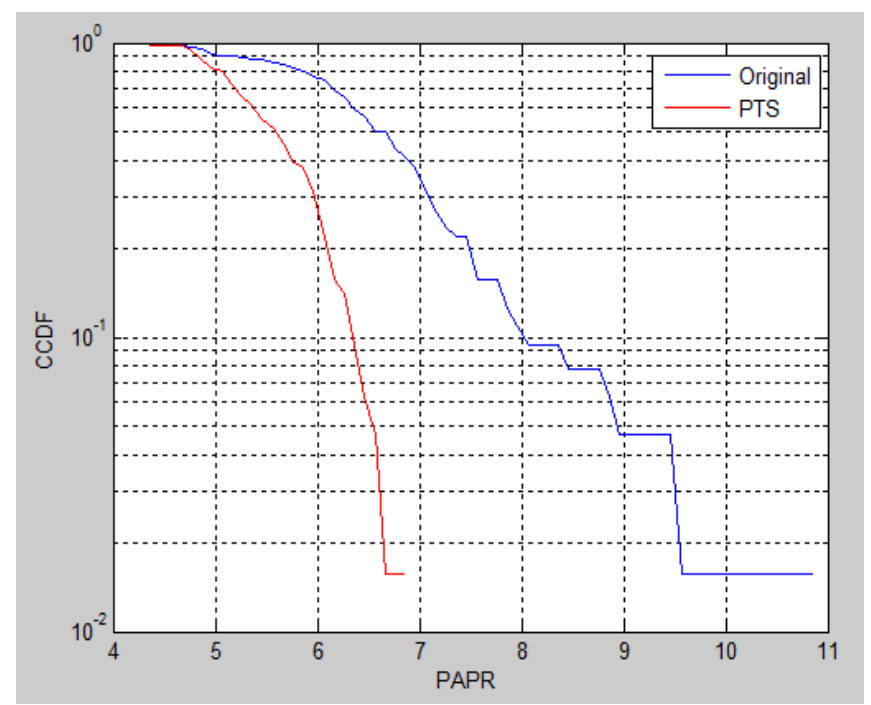

\section{CONCLUSION}

Partial transmit sequence (PTS) is commonly used peak to average power ratio (PAPR) reduction technique in OFDM system without any distortion and to reduce the computational complexity of PTS we introduce parallel and serial PTS method based on the fact that the weighting factors as chosen as code words of linear block code. Parallel and serial PTS also provide error correction capability for the weighting factors.

\section{REFERENCES}

[1] Shilpa Sharma, pradeep Kumar gaur, "Survey on PAPR Reduction Techniques in OFDM System," International Journal Of Advanced Research In Computer And Communication Engineering, Vol. 4, Issue 6, June 2015.

[2] Pooria Varahram, Borhanuddin Mohd Ali, "A Low Complexity Partial Transmit Sequence for Peak to Average Power Reduction in OFDM System," Radio engineering, Vol. 20.No. 3, September 2011.

[3] Malhar Chauhan, Saurabh patel, Hardik patel, "Different Techniques to Reduce the PAPR in OFDM System," International Journal of Engineering Research and Applications, Vol. 2, Issue 3, May-Jun 2012.

[4] Dae-Woon Lim, Seok-Joong Heo, Jong-Seon No, and Habong Chung, Member, IEEE," A New PTS OFDM Scheme with Low Complexity for PAPR Reduction", IEEE Transactions on Broadcasting, Vol. 52, No. 1, March 2006.

[5] Zeyid T. Ibraheem, Md. Mijanur Rahman, S. N. Yaakob, Mohammad Shahrazel Razalli, and Kawakib K. Ahmed, "Performance Analysis of a Combined PTS Partitioning Scheme for PAPR Reduction in OFDM Signals under Different Modulation Techniques", International Journal of Engineering and Technology (IJET) Vol 6 No 6 Dec 2014Jan 2015.

\section{BIOGRAPHIES}

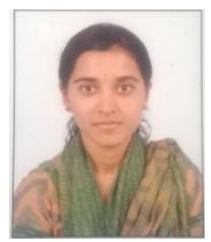

Ms. Amrutha $\mathbf{P}$ is pursuing M.Tech degree in Digital Communication and Networking from T. John Institute of Technology, Bengaluru afflicted to Visvesvaraya Technological University.

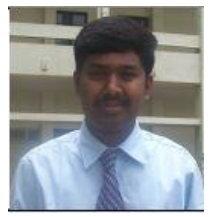

Mr. Anandswamy A S is the Associate Professor of Electronics and Communication Engineering, T. John Institute of Technology, Bengaluru. 